\title{
Existence Theorems of Permanent Gravity Waves on Water of Uniform Depth
}

\author{
By
}

\author{
Masako Kumazawa*
}

\section{Preface}

As for gravity waves of permanent type on water surface, existence theorems have been presented by many authors since Lévi-Cività (1925). But yet it seems to have no simple proof which covers all cases. On the other hand, the calculation scheme for practical computation of these waves has been unified in a simple formula $([1])$, and it has already been shown that the result of the approximate calculation is in a good agreement with experimental fact $([2])$.

This unified calculation formula is based on a formulation to determine a holomorphic function in a unit circle with some nonlinear boundary condition on the circumference, and in the case of infinite water depth it just agrees with Lévi-Cività's formulation itself. Hence we want to extend the proof of the latter to our general case. But as for the case of solitary wave, i.e., the case of infinite wave length, and of the highest wave, i.e., the case having angular crests, the asking holomorphic function has the corresponding singular point on the unit circle, so it is difficult formally to extend, and our effort is yet unsuccessful. Therefore in the following we deal with the case of the periodic wave having round crests and our method is based on extending directly Herbert Beckert's proof ([3]) concerning with the case of infinite depth to our case.

The author wishes to acknowledge her indebtedness to Hikoji Yamada for suggesting this problem and also to Reiko Sakamoto, under whose

Received June 22, 1970.

Communicated by S. Matsuura.

* Nara Women's University. 
direction this paper has been accomplished.

\section{Problem}

As for the transformation onto a unit circle, we refer to [1] and summarize it in the following:

We treat permanent gravity waves on water of uniform depth. And we suppose that our waves are periodic and are symmetric about the vertical line through the crest and observe the waves on the water from the coordinates system $O-x y$ which follows after the waves as fast as the waves, so that the wave form stands fixed relative to the axes, and water flows steadily from left to right (say). The origin $O$ is at one wave crest, the $x$-axis is horizontal and directed to right, the $y$-axis vertical and upward. We consider a water region $\mathrm{ABCOC}^{\prime} \mathrm{B}^{\prime}$ (Fig. $a$ ).

Let

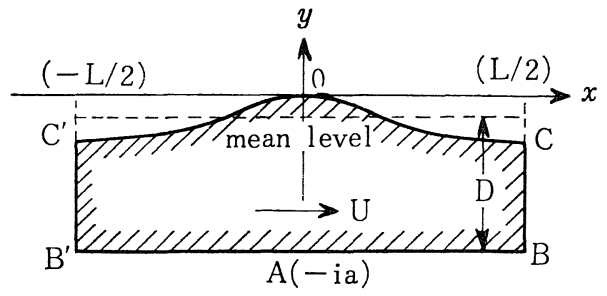

Fig. $a$ Z $-p l$

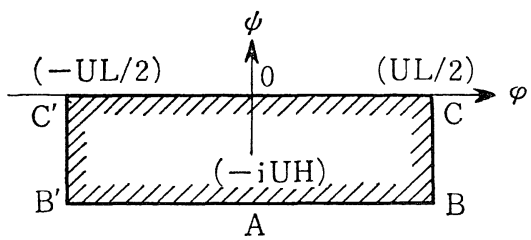

Fig. $b \quad W-p l$

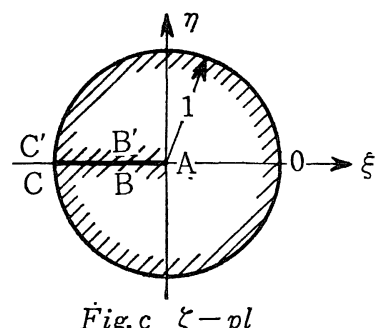

Fig. $c \zeta-p l$

$$
\begin{array}{r}
W(z)=\varphi(x, y)+i \psi(x, y), \\
z=x+i y
\end{array}
$$

be the complex potential function in which $\varphi$ is the potential function and $\phi$ is the stream function and arbitrary constant is fixed so that $W(0)=0$. Complex velocity is given by

$$
\frac{d W}{d z}=u(x, y)-i v(x, y)
$$

where $u$ and $v$ are the velocity components and

$$
\varphi_{x}=\phi_{y}=u, \varphi_{y}=-\psi_{x}=v,
$$

and $u, v$ are periodic with respect to $x$. Let the wave-length be denoted by $L$ and the mean depth 
by $D$. We define the wave velocity $U$ by the formula:

$$
U L=\int_{-L / 2}^{L / 2} u(x, y) d x=\varphi\left(\frac{L}{2}, y\right)-\varphi\left(-\frac{L}{2}, y\right)
$$

which is independent of $y$ because of our wave form.

When we denote

$$
\frac{d W}{d z}=|\nu| e^{-i 0}
$$

$|\nu|$ and $\theta$ are the speed and the direction of flowing water, $\theta$ being the angle measured upwards from the horizontal direction (to right). Denoting $|\nu| / U$ by $q$ and $\log q$ by $\tau, 5)$ becomes to

$$
\frac{1}{U} \frac{d W}{d z}=q e^{-i \theta}=e^{-i \Omega(z)}
$$

the field quantity $\Omega(z)$ being defined by the relation:

$$
\Omega(z)=\theta+i \tau=i \log \left(\frac{1}{U} \frac{d W}{d z}\right)
$$

Evidently $\Omega(z)$ is holomorphic at every interior point of the water region, and may have singular points at the boundary of the region.

Along the streamline which constitutes the free surface of water a constant atmospheric pressure prevails, and by Bernoulli's equation we have

$$
q^{2}+\frac{2 g}{U^{2}} y=\mathrm{const}
$$

or differentiating this along the arc $s$ of the stream line we have

$$
q \frac{d q}{d s}+\frac{g}{U^{2}} \sin \theta=0
$$

where $\theta$ is the inclination of free surface. Moreover we have

$$
\psi(x, y)=0
$$

along the streamline and put

$$
\psi(x,-a)=-U H
$$




$$
\varphi\left(\frac{L}{2}, y\right)=\frac{U L}{2}, \quad \varphi\left(-\frac{L}{2}, y\right)=-\frac{U L}{2}
$$

And because of our wave form, it takes the horizontal velocity at points under crest or trough, or at the bottom, i.e.,

$$
v(0, y)=v\left( \pm \frac{L}{2}, y\right)=v(x,-a)=0
$$

The complex potential function $W(z)$ maps the physical $z$-pl. onto the $W$-pl. as shown in Fig. $b$.

Now we introduce the complete elliptic integral $K(k)$ and $K\left(k^{\prime}\right)$, of the first kind with the modulus $k$ and its complementary modulus $k^{\prime}$ $=\sqrt{1-k^{2}}$, and define the numerical value of $k$ by the relation

$$
\frac{K^{\prime}}{K}=2 \frac{H}{L} \text {. }
$$

With $k, k^{\prime}, K$ and $K^{\prime}$ thus determined we define a transformation

$$
s n\left(\frac{2 K}{U L} W, k\right)=-i \frac{1-\zeta}{2 \sqrt{\zeta}},
$$

where $s n$ is the Jacobian elliptic function with modulus $k$, which maps the $W$-pl. onto $\zeta$-pl. By this mapping the one wave length region of the $W$-pl., which is shown in Fig. $b$, corresponds to the region interior to the unit circle with cut about the origin of the $\zeta$-pl. also shown in Fig. c. From $5^{\prime}$ ) and 10) we have

$$
d z=i \frac{L}{4 K} \frac{1}{\sqrt{\zeta^{2}+\frac{k^{2}}{4} \zeta(1-\zeta)^{2}}} e^{i \Omega(\zeta)} d \zeta
$$

where $\Omega(\zeta)$ is the transform of $\Omega(z)$ on $\zeta$-pl. It is verified that $-i \Omega(\zeta)$ has a pair of complex conjugate values at every pair of conjugate points in the unit circle $|\zeta| \leqq 1$ and it takes real value on the real axis and all along the cut $-1 \leq \zeta<0$ values of the two sides coincide. Thus the function $-i \Omega(\zeta)$ and consequently $\Omega(\zeta)$ itself is not only holomorphic in the cut unit circle, but also in the unit circle without cut.

When we take $d z$ along the free surface this is equal to $d s e^{i \theta}$ and 
has to correspond to $d \zeta=i e^{i \sigma} d \sigma$ on the unit circle $\zeta=e^{i \sigma}$, $\sigma$ being the arc length of the unit circle. The correspondence is given by 11) and by use of the expression $5^{\prime}$ ) we have

$$
d s=-\frac{L}{4 K} \frac{d \sigma}{\sqrt{1-k^{2} \sin ^{2} \frac{\sigma}{2}}} .
$$

The boundary condition 8 ) is transformed on the unit circle $|\zeta|=1$ in the $\zeta$-pl. as follows:

$$
q^{2} \frac{d q}{d \sigma}=p \frac{\sin \theta(\sigma)}{\sqrt{1-k^{2} \sin ^{2} \frac{\sigma}{2}}}
$$

or

$$
\frac{d \tau}{d \sigma}=\frac{p e^{-3 \tau(\sigma)} \sin \theta(\sigma)}{\sqrt{1-k^{2} \sin ^{2} \frac{\sigma}{2}}}
$$

where $p$ is defined by

$$
p=\frac{g L}{4 K U^{2}}=\frac{g H}{2 K^{\prime} U^{2}}
$$

13) $\left(13^{\prime}\right)$ ) is the boundary condition for the determination of $\Omega(\zeta)$, and $p$ is the eigenvalue which has to be determined simultaneously with $\Omega(\zeta)$. From $p$ the wave velocity $U$ follows at once.

Thus our mathematical formulation is completed and Stokes' waves and the solitary wave are easily seen to be the two extreme cases $(k=0$ and $k=1$ ) of our present formulation.

Now our problem is reduced to the following:

Find a function

$$
\begin{aligned}
& \Omega(\zeta)=\Theta(\zeta)+i T(\zeta) \\
& (\Theta(\zeta)=\operatorname{Re} \Omega(\zeta), \quad T(\zeta)=\operatorname{Im} \Omega(\zeta))
\end{aligned}
$$

which is holomorphic in the unit circle $C=\{|\zeta| \leq 1\}$, denoting

$$
\Omega(\zeta)=\Omega\left(\rho e^{i \sigma}\right)=\Omega(\rho, \sigma)
$$


$\Omega(\rho, \sigma)$ is continuously differentiable in $\tilde{C}=\{0 \leq \rho \leq 1,-\pi \leq \sigma \leq \pi\}$, and moreover satisfies the following properties:

a) Denoting the restriction of $\Omega(\zeta)$ on $S=\{|\zeta|=1\}$ by

$$
\Omega\left(e^{i \sigma}\right)=\Omega(1, \sigma)=\omega(\sigma)=\theta(\sigma)+i \tau(\sigma),
$$

$\theta(\sigma)$ and $\tau(\sigma)$ satisfy the relation:

$$
\frac{d \tau(\sigma)}{d \sigma}=\frac{p e^{-3 \tau(\sigma)} \sin \theta(\sigma)}{\sqrt{1-k^{2} \sin ^{2} \frac{\sigma}{2}}},
$$

where $k$ is a given constant $(0 \leq k<1)$, and $p$ is some positive constant (arbitrary).

b) $\Theta(\bar{\zeta})=-\Theta(\zeta)$

c) 1) $|\Theta(\zeta)| \leq \beta<\frac{\pi}{2} \quad(\beta$ is a given positive constant $)$

2) $\Theta(\zeta) \geq 0 \quad$ for $\operatorname{Im} \zeta \geq 0$

d) $\frac{T(1)}{T(-1)}=\gamma \quad(\gamma$ is a given constant $0<\gamma<1)$.

\section{Proof of Existence}

Let $\mathscr{B}_{\nu}(0<\nu<1)$ be the Banach space which consists of functions $f$ defined on $S$, which is represented by $f=f(\sigma)(-\pi \leq \sigma \leq \pi)$, with the norm defined by

$$
\|f\|_{\nu}=\max _{-\pi \leq \sigma \leq \pi}|f(\sigma)|+H_{\nu}(f)
$$

where $H_{\nu}(f)$ is the Hölder constant of $f$, i.e.,

$$
H_{\nu}(f)=\sup _{-\pi \leq \sigma, \sigma^{\prime} \leq \pi} \frac{\left|f\left(\sigma^{\prime}\right)-f(\sigma)\right|}{\left|\sigma^{\prime}-\sigma\right|^{\nu}} .
$$

And $\mathscr{B}_{k+\nu}(0<\nu<1, k=0,1,2, \ldots)$ is defined by

$$
\mathscr{B}_{k+\nu}=\left\{f ; f, f^{\prime}, \ldots, f^{(k)} \in \mathscr{B}_{\nu}\right\}
$$

with the norm 


$$
\|f\|_{k+\nu}=\max _{-\pi \leq \sigma \leq \pi}|f(\sigma)|+\cdots+\max _{-\pi \leq \sigma \leq \pi}\left|f^{(k)}(\sigma)\right|+H_{\nu}\left(f^{(k)}(\sigma)\right) .
$$

In the following, we fix some $\nu(0<\nu<1)$ and consider subsets of $\mathscr{B}_{\nu}$, namely $Q$, which consists of functions satisfying following relations
1) $\theta(-\sigma)=-\theta(\sigma)$
2) $\theta(\sigma) \geq 0 \quad$ for $0 \leq \sigma \leq \pi$

and $\tilde{Q}$, which is included in $Q$ and functions in $\tilde{Q}$ satisfy the inequality

3) $|\theta(\sigma)| \leq \beta<\frac{\pi}{2}$.

Now taking some $\theta \in \tilde{Q}$ and putting it into (1), we can consider (1) as the differential equation of $\tau=\tau(\sigma)$ and integrating it, we get the equation

$$
e^{3 \tau(\sigma)}=e^{3 \tau(0)}+3 p \int_{0}^{\sigma} \frac{\sin \theta(\sigma)}{\sqrt{1-k^{2} \sin ^{2} \frac{\sigma}{2}}} d \sigma
$$

In the above equation, the integral constant $e^{3 \tau(0)}$ should be connected with the condition $\mathrm{d}$ ), but now we suppose $e^{3 \tau(0)}=1$ and modify it later. Hence we get

$$
\tau(\sigma)=\frac{1}{3} \log (1+F(\sigma))
$$

where

$$
F(\sigma)=3 p \int_{0}^{\sigma} \frac{\sin \theta(\sigma)}{\sqrt{1-k^{2} \sin ^{2} \frac{\sigma}{2}}} d \sigma
$$

Moreover we have

$$
\frac{d \tau}{d \sigma}=\frac{p \sin \theta(\sigma)}{(1+F(\sigma)) \sqrt{1-k^{2} \sin ^{2} \frac{\sigma}{2}}} .
$$

Let $T(\rho, \sigma)$ be harmonic function in $\widetilde{C}$ with the boundary value $\tau(\sigma)$ defined by (3), and $\Omega^{*}(\rho, \sigma)=\Theta^{*}(\rho, \sigma)+i T(\rho, \sigma)$ be the holomorphic function in $\tilde{C}$ whose imaginary part is $T(\rho, \sigma)$ and $\Theta^{*}(0,0)=0$. Denote 
the boundary value of $\Theta^{*}(\rho, \sigma)$ by $\theta^{*}=\theta^{*}(\sigma)$, which is the conjugate function of $\tau(\sigma)$. We consider a mapping $T_{p}$ which maps $\theta(\sigma) \in \tilde{Q}$ to $\theta^{*}(\sigma)$. Now our problem is reduced to looking for a fixed point of $T_{p}$. Then it comes into question whether we may find a subset of $\tilde{Q}$ which satisfies the condition of Schauder's fixed point theorem, namely whether there may be a closed convex set in $Q$ which $T_{p}$ maps into itself continuously and completely continuously. Although $\theta(\sigma) \equiv 0$ is one of fixed points, we want to show that there are nontrivial fixed points in the following. Our proof is based on the following two theorems about conjugate functions :

Theorem (Privaloff). If $f \in \mathscr{B}_{k+\nu}$, then $\bar{f} \in \mathscr{B}_{k+\nu}$, where $\bar{f}$ is the conjugate function of $f$. And there exists a positive constant $C_{k+\nu}$ depending only on $\nu$ and $k$, such that

$$
\|\bar{f}\|_{k+\nu} \leq C_{k+\nu}\|f\|_{k+\nu}
$$

where $\bar{f}(0)=0$.

Theorem (Beckert) (cf. [3] pp. 382-383). If $f \in \mathscr{B}_{\nu}$ is continuously differentiable and

$$
\begin{aligned}
& f(\sigma)=f(-\sigma) \\
& \frac{d f}{d \sigma}(\sigma) \geq 0 \quad \text { for } 0 \leq \sigma \leq \pi
\end{aligned}
$$

then

$$
\begin{aligned}
& \bar{f}(-\sigma)=-\bar{f}(\sigma) \\
& \bar{f}(\sigma) \geq 0 \quad \text { for } \quad 0 \leq \sigma \leq \pi
\end{aligned}
$$

where $\bar{f}$ is the conjugate function of $f$. And if $\frac{d f}{d \sigma}\left(\sigma_{0}\right)>0$ for $0 \leq \sigma_{0}$ $\leq \pi$, then $\bar{f}\left(\sigma_{0}\right)>0$.

Now we fix some $p_{0}$ such that

$$
p_{0}>\frac{\pi}{2}
$$


and consider $p$ satisfying $0<p \leq p_{0}$. We also fix $k_{0}$ as $0<k_{0}<\frac{\pi}{2}$ and consider $k$ satisfying $0 \leq k \leq k_{0}$. We remark the uniformity with respect to $k$ throughout in the following.

Lemma 1. The image $\theta^{*}(\sigma)$ of $\theta(\sigma) \in \tilde{Q}$ with respect to mapping $T_{p}$ belongs to $Q$ and there exists a positive constant $K$ which depends only on $\nu, p_{0}$ and $k_{0}$, such that $\theta^{*}(\sigma)$ is included in the sphere

$$
I_{K}:\left\|\theta^{*}\right\|_{\nu} \leq K
$$

and

$$
\left\|\theta^{*}\right\|_{1+\nu} \leq K_{1}\|\theta\|_{\nu}
$$

where $K_{1}$ is also a positive constant depending on $\nu, p_{0}$ and $k_{0}$.

First of all, we get the following estimation from $\left(3^{\prime}\right)$

$$
\left|\frac{d \tau}{d \sigma}\right| \leq \frac{p}{\sqrt{1-k^{2}}}
$$

In fact, since $\theta \in \tilde{Q}$, it holds $F(\sigma) \geq 0$ in the interval $-\pi \leq \sigma \leq \pi$. Then $T_{p} \theta \in I_{K}$ is shown by Privaloff's theorem. It follows from Beckert's theorem that $T_{p} \theta \in Q$. Next we have

$$
\|\tau\|_{1+\nu} \leq \frac{2 \pi p_{0}\left(1+\left(1+3 p_{0}\right)(2 \pi)^{-\nu}\right)}{\left(1-k_{0}^{2}\right)^{\frac{3}{2}}}\|\theta\|_{\nu}
$$

then

$$
\left\|\theta^{*}\right\|_{1+\nu} \leq \frac{2 \pi C_{\nu} p_{0}\left(1+\left(1+3 p_{0}\right)(2 \pi)^{-\nu}\right)}{\left(1-k_{0}^{2}\right)^{\frac{3}{2}}}\|\theta\|_{\nu}
$$

by Privaloff's theorem, where $C_{\nu}$ is a constant depending on $\nu$.

It has been shown that the image of $\tilde{Q}$ with respect to $T_{p}$ is included in $Q$, but generally it is not included in $\tilde{Q}$. So in $Q$, we consider a family of convex sets $Q_{s}$ with a parameter $s$ which is characterized by

$$
Q_{s}=\left\{\theta(\sigma) \in Q ; \frac{1}{\pi} \int_{-\pi}^{\pi} \theta(\sigma) \sin \sigma d \sigma=s\right\}
$$

Hence $Q=\bigcup_{s \geq 0} Q_{s}$. Now let $M_{s}$ be the intersection of $Q_{s}$ and $I_{K}$, then 
$Q \cap I_{K}=\bigcup_{s \geq 0} M_{s}$

Lemma 2. There exists a positive constant $\eta$ which depends only on $\nu, p_{0}$ and $k_{0}$ such that, if $\theta(\sigma) \in M_{s}(0 \leq s \leq \eta)$, then

$$
|\theta(\sigma)| \leq \beta<\frac{\pi}{2}
$$

namely

$$
\bigcup_{0 \leq s \leq \eta} M_{s} \subset \tilde{Q}
$$

Here we consider another mapping $T_{p}{ }^{\prime}$. Namely, for $\theta \in Q$ integrating the equation

$$
\frac{d \tau^{\prime}}{d \sigma}=p \theta(\sigma)
$$

we have $\tau^{\prime}(\sigma)$ with $\tau^{\prime}(0)=0$ and let $\theta^{* \prime}(\sigma)$ be the conjugate function of $\tau^{\prime}(\sigma)$ and define $T_{p}^{\prime}$ by

$$
T_{p}^{\prime} \theta(\sigma)=\theta^{* \prime}(\sigma)
$$

For mapping $T_{p}{ }^{\prime}$, we have the following lemma (cf. [3] p. 385).

Lemma 3. If $\theta(\sigma) \in Q_{s}$, then $T_{p}^{\prime} \theta=\theta^{* \prime}(\sigma) \in Q_{p s}$.

Comparing $T_{p}$ and $T_{p}^{\prime}$, we get

Lemma 4. Let $\theta \in \underset{0<s \leq \eta}{\bigcup} M_{s}$, then $T_{p} \theta \in M_{s^{*}(p, \theta)}$, where $s^{*}$ has following properties:

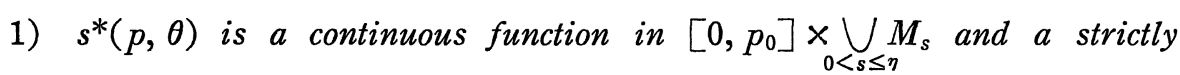
increasing function of $p$.

2) There exists a positive number $\lambda$ depending only on $\nu, p_{0}$ and $k_{0}$, such that for $\theta \in M_{s}(0<s \leq \min (\lambda, \eta))$ it holds that

$$
s^{*}\left(p_{0}, \theta\right) \geq s
$$

3) If $p \leq \sqrt{1-k^{2}}$, then it holds that

$$
s^{*}(p, \theta) \leq s \quad \text { for } \theta \in M_{s}
$$


It is verified by Privaloff's theorem that if $\theta_{j}(\sigma) \underset{j \rightarrow \infty}{\longrightarrow} \theta(\sigma)$ in $\mathscr{B}_{\nu}$ and $p_{j} \underset{j \rightarrow \infty}{\longrightarrow} p$, then $T_{p_{j}} \theta_{j}(\sigma) \underset{j \rightarrow \infty}{\longrightarrow} T_{p} \theta(\sigma)$ in $\mathscr{B}_{\nu}$, namely $T_{p} \theta$ is a continuous with respect to $(p, \theta)$. Then $s^{*}(p, \theta)$ is a continuous function of $(p, \theta)$. And applying Beckert's theorem to the difference between two different values of $p$, we have the monotonicity with respect to $p$. To show 2) and 3 ), we use

$$
\frac{2}{\pi} \theta \leq \sin \theta \leq \theta, \quad 0 \leq \theta \leq \frac{\pi}{2} .
$$

And when we put

$$
\int_{0}^{\pi} \sin \theta(\sigma) d \sigma=t
$$

for $\theta(\sigma) \in M_{s}(0<s \leq \eta)$, we get inequality

$$
\frac{\frac{2}{\pi} p_{0} \theta(\sigma)}{1+\frac{3 p_{0} t}{\sqrt{1-k^{2}}}} \leq \frac{d \tau}{d \sigma} \leq \frac{p_{0} \theta(\sigma)}{\sqrt{1-k^{2}}}, \quad 0 \leq \sigma \leq \pi
$$

Now putting

$$
p_{0}^{\prime}=\frac{\frac{2}{\pi} p_{0}}{1+\frac{3 p_{0} t}{\sqrt{1-k^{2}}}}
$$

we denote

$$
T_{p_{0}} \theta=\theta^{*} ; \quad T_{p_{0}{ }^{\prime}} \theta=\theta^{* \prime}
$$

So it is shown that

$$
\theta^{*} \in M_{s^{*}}, \quad \theta^{* \prime} \in Q_{p_{0}{ }^{\prime} s}
$$

and applying Beckert's theorem to $\theta^{*}-\theta^{* \prime}$, we get

$$
s^{*} \geq p_{0}{ }^{\prime} s
$$

Hence if $p_{0}{ }^{\prime} \geq 1$, then $s^{*} \geq s$. In order that it holds 


$$
p_{0}{ }^{\prime}=\frac{\frac{2}{\pi} p_{0}}{1+\frac{3 p_{0} t}{\sqrt{1-k^{2}}}} \geq 1
$$

$t$ must satisfy

$$
l=\frac{\sqrt{1-k^{2}}}{3}\left(\frac{2}{\pi}-\frac{1}{p_{0}}\right) \geq t
$$

Since $p_{0}>\frac{\pi}{2}, l>0$, therefore there exists a positive number $\lambda$ similarly to Lemma 2, such that for $0<s \leq \lambda$, every $\theta \in M_{s}$ satisfies the condition (11). Consequently $T_{p_{0}}$ maps $M_{s}(0<s \leq \min (\lambda, \eta))$ into $\underset{s^{*} \geq s}{\bigcup} M_{s^{*}}$. Similarly to show 3$)$, it is sufficient to compare $T_{p}$ with $T_{p^{\prime \prime}}\left(p^{\prime \prime}=\frac{p}{\sqrt{1-k^{2}}}\right)$.

Corollary of Lemma 4. For each $\theta \in M_{s}(0<s \leq \min (\lambda, \eta))$, there exists a positive number $p_{\theta}\left(\sqrt{1-k^{2}} \leq p_{\theta} \leq p_{0}\right)$ such as to satisfy

$$
T_{p} \theta \in M_{s}
$$

and $p_{\theta}$ is continuous with respect to $\theta$. Then, for $\theta \in M_{s}$, putting

$$
T \theta=T_{p_{\theta}} \theta
$$

$T$ is a mapping from $M_{s}$ into $M_{s}$ for each $s(0<s \leq \min (\lambda, \eta))$.

Lemma 5. $T$ is continuous and completely continuous.

If $\|\theta\|_{\nu} \leq 1$, then we have $\left\|\theta^{*}\right\|_{1+\nu} \leq K_{1}$ by Lemma 1 . So complete continuity is led by Ascoli-Arzela's theorem. Next since $p_{\theta}$ is continuous with respect to $\theta$ and $T_{p} \theta$ is continuous with respect to $(p, \theta)$, we have $T_{p_{\theta}} \theta_{j} \underset{j \rightarrow \infty}{\longrightarrow} T_{p_{\theta}} \theta$ in $\mathscr{B}_{\nu}$. Consequently $T$ is continuous.

As we have seen, the assumption of Schauder's fixed point theorem has been assured, so the transformation $T$ in $M_{s}(0<s \leq \min (\lambda, \eta))$ has at least one fixed point which belongs to $\mathscr{B}_{1+\nu}$ by Lemma 1 . The fixed point $\theta_{0}$ is the restriction to $S$ of the real part of holomorphic function $\Omega_{0}(\zeta)=\Theta_{0}(\zeta)+i T_{0}(\zeta)$ in $C$ which satisfies the boundary condition (1) for $p=p_{\theta_{0}}\left(p_{0} \geq p_{\theta_{0}} \geq \sqrt{1-k^{2}}\right)$. Moreover since $\theta_{0}(-\sigma)=-\theta_{0}(\sigma)$, then $\Theta_{0}(\sigma)$ satisfies the condition $b)$. And from 


$$
\begin{aligned}
& \left|\theta_{0}(\sigma)\right| \leq \beta<\frac{\pi}{2} \\
& \theta_{0}(\sigma) \geq 0 \quad \text { for } 0 \leq \sigma \leq \pi
\end{aligned}
$$

$\Theta_{0}(\sigma)$ satisfies the condition $\mathrm{c}$ ) by the maximum principle. But the condition d), i.e.,

$$
\frac{\tau(0)}{\tau(\pi)}=\gamma, \quad 0<\gamma<1
$$

is not satisfied in general. So now putting

$$
\tau(\sigma)=\tau_{0}(\sigma)+C
$$

and deciding a constant $C$ as to satisfy the above equality, we have

$$
C=\frac{\gamma \tau_{0}(\pi)}{1-\gamma}
$$

\section{Conclusion}

Fixing $p_{0}>\frac{\pi}{2}$ and $0<k_{0}<\frac{\pi}{2}$ arbitrarily, there exist two positive numbers $\eta$ and $\lambda$ which depend only on $\nu, p_{0}$ and $k_{0}$. And for each $s, 0<s \leq \min (\lambda, \eta)$, there exists $\theta_{0}(\sigma) \in M_{s}$ and a positive number $p_{\theta_{0}}$ $\left(p_{0} \geq p_{\theta_{0}} \geq \sqrt{1-k^{2}}\right)$ as follows. Denote

$$
\omega(\sigma)=\theta(\sigma)+i \tau(\sigma)
$$

where

$$
\begin{aligned}
& \theta(\sigma)=\theta_{0}(\sigma) \\
& \tau(\sigma)=\tau_{0}(\sigma)+C
\end{aligned}
$$

and

$$
\begin{gathered}
\tau_{0}(\sigma)=\frac{1}{3} \log \left(1+3 p_{\theta_{0}} \int_{0}^{\sigma} \frac{\sin \theta_{0}(\sigma)}{\sqrt{1-k^{2} \sin ^{2} \frac{\sigma}{2}}} d \sigma\right) \\
C=\frac{\gamma \tau_{0}(\pi)}{1-\gamma}
\end{gathered}
$$


then $\theta(\sigma)$ and $\tau(\sigma)$ are continuously differentiable functions on $S$ satisfying the relation

$$
\frac{d \tau(\sigma)}{d \sigma}=\frac{p e^{-3 \tau(\sigma)} \sin \theta(\sigma)}{\sqrt{1-k^{2} \sin ^{2} \frac{\sigma}{2}}}, \quad p=p_{\theta_{0}} e^{3 c}
$$

Let $\Omega(\zeta)$ be a holomorphic function in $C$ whose boundary value $\Omega\left(e^{i \sigma}\right)$ is $\omega(\sigma)$, i.e.,

$$
\Omega(\zeta)=\frac{1}{2 \pi i} \int_{-\pi}^{\pi} \frac{\omega(\sigma)}{e^{i \sigma}-\zeta} d \sigma
$$

then $\Omega(\zeta)$ is one of which we ask for.

\section{References}

[1] Yamada, H., Permanent gravity waves on water of uniform depth, Reports of Res. Inst. Appl. Mech. Kyushu Univ., Vol. VI, No. 23, 1958.

[2] Yamada, H. and Tadahiko Shiotani, On the highest waves of permanent type, Bull. Disas. Priv. Res. Inst. Kyoto Univ., Vol. 18, Part 2, 1968.

[3] Beckert, H., Existenzbeweise in der Theorie permanenter Schwerewellen einer inkompressiblen Flüssigkeit längs eines Kanals, Arch. Rational Mech. Anal. 9 (1962), 379-394. 